\title{
Wideband UHF and SHF long-range channel characterization
}

\author{
Edward Kassem ${ }^{1}$, Jiri Blumenstein ${ }^{1 *}$ (D), Ales Povalac ${ }^{1}$, Josef Vychodil ${ }^{1}$, Martin Pospisil ${ }^{1}$, Roman Marsalek $^{1}$ \\ and Jiri Hruska ${ }^{2}$
}

\begin{abstract}
This paper presents an outdoor long-range (from $315 \mathrm{~m}$ up to $5.3 \mathrm{~km}$ ) fixed channel campaign for both ultra high frequency and super high frequency bands with co-polarized horizontal and vertical antenna configurations. It investigates the channel characteristics of device to device communication scenarios underlaying the 5th generation networks by providing detailed research. Both line of sight and non-line of sight measurements in $1.3 \mathrm{GHz}$ and $5.8 \mathrm{GHz}$ frequency bands with bandwidth up to $600 \mathrm{MHz}$ were conducted. The path loss, root mean square delay spread, coherence bandwidth, and channel frequency response variation are characterized. We observed that the variation is negligible in microcell line of sight environment for both above mentioned frequencies, whereas it significantly increases with frequency in different macrocell non-line of sight environments. The distance dependency of path loss was also derived. It was observed that the root mean square delay spread decreases with frequency for both line of sight microcell and non-line of sight macrocell measurements. A dependency between the root mean square delay spread and transmitter-receiver distance in non-line of sight environments was also captured. The relation between the coherence bandwidth and the root mean square delay spread was depicted. It demonstrates an exponential function in all considered channel combinations.
\end{abstract}

Keywords: Channel model, Device to device, Line of sight, Non-line of sight, Super high frequency, Ultra high frequency

\section{Introduction}

Device to device communication [1,2] is an important technology which enables data flow not only between humans but also between machines without human intervention. It can be used underlying the available cellular networks. The 5th generation system technology, 3rd Generation Partnership Project Release 15, will have to support high performance in spectral efficiency and throughput measurements. The 5 th generation network is one of the most suitable environments for device to device communication since it is an IP-based network that enables to control any connected devices using internet protocols. Moreover, it is able to send large amounts of data with a high rate and low latency and support a large amount of connected devices. It is a good solution to reduce the eNB traffic load and the end to end delay. In

*Correspondence: blumenstein@feec.vutbr.cz

${ }^{1}$ Department of Radio electronics, Brno University of Technology, Technicka

12, Brno, Czech Republic

Full list of author information is available at the end of the article order to develop a reliable wireless device to device communication network [3, 4], an accurate description of the wireless channel impulse response measurements should be presented. The channel impulse response describes spreading, echoing, multipath propagation, and Doppler effects that occur when an impulse is sent between the transmitter and the receiver. Knowledge of the channel impulse response characteristics enables system designers to ensure that inter symbol interference does not dominate and hence lead to an excessive irreducible bit error ratio [5].

\subsection{Literature review}

As mentioned above, propagation measurements are necessary for creating statistical channel models that support the development of new standards and technologies for wireless communications systems. Channel models that predict signal strength and multipath time delays are required for a proper system design. There have been a number of studies for channel sounding using different input signals over the past 10 years. 
As a sample of typical work, there is a paper which studied the frequency dependence of the channel characteristics at the $2-4 \mathrm{GHz}$ frequency band [6]. Line of sight and obstructed line of sight scenarios were considered. Angle of arrival and delay of arrival of the main paths were investigated. A rich multipath environment was observed, with intensive path components existence in both angle and delay domains.

Outdoor measurements were conducted in an open-area test site at the National Metrology Institute of Germany [7], to study the scattering effects of a traffic sign on vehicles moving along the road. The outputs are analytical modeling, simulation, measurement, and implementation of the bi-static radar cross section of the traffic signs.

A paper on outdoor sounding [8] highlighted the propagation path loss models for 5 th generation urban micro and macro cellular scenarios. It compares the alphabeta-gamma and the close-in free space reference distance models. A wide range of frequencies $2-73.5 \mathrm{GHz}$ over 5-1429 $\mathrm{m}$ distances were used. The output showed very comparable modeling performance between closein and alpha-beta-gamma models. The close-in model offers simplicity and a conservative non-line of sight path loss estimate at large distances, whereas the alpha-betagamma model is more complex and offers a fraction of a decibel smaller shadow, less loss near the transmitter, and more loss far from transmitter.

Another paper [9] described the achieved results for line of sight and non-line of sight measurements between the User Equipment and the base station in Nanjing Road, Shanghai. The received signals were $20 \mathrm{MHz}$ bandwidth with $2.1376 \mathrm{GHz}$ carrier frequency. The delays and the complex attenuations of multipath components have been estimated by applying the space-alternating generalized expectation-maximization algorithm. The distance between transmitter and receiver in line of sight/nonline of sight scenarios, the life-distance of the line of sight channel, the power variation at line of sight to nonline of sight transition, and the transition duration were extracted.

The authors in [10] presented a sounding system that uses an orthogonal frequency division multiplexing signal at $5.6 \mathrm{GHz}$ with $200 \mathrm{MHz}$ bandwidth. The power delay profiles and the excess delay were presented.

An open-pit mine campaign performed a $25-\mathrm{MHz}$ wide frequency band sounding immediately below the unlicensed 2.4-GHz ISM band [11]. A continuously repeating maximum-length or $\mathrm{m}$-sequence with $K=2047$ sequence length was adopted as a transmitted signal. It was transmitted at a rate of $25 \mathrm{MS} / \mathrm{s}$. Four measurement realizations of the impulse response with different transmitter-receiver separations that vary between 425$1670 \mathrm{~m}$ were recorded. The calculated delay spread of the channel was often more than $10 \mu s$.
A channel measurement campaign was conducted to study the frequency dependence of the propagation channel for a wide range of frequencies $3-18 \mathrm{GHz}$ [12]. Urban macro and micro cellular environments were covered. The root mean square delay spreads, coherence bandwidth, path loss, shadow fading, and Ricean factor were characterized. It is mentioned that the path loss exponents vary significantly with frequency (from 1.8 to $2 \mathrm{~dB}$ in a line of sight environment and from 2.71 to $4.34 \mathrm{~dB}$ in non-line of sight). Shadow fading and the Ricean factor increase with frequency, whereas the root mean square delay spread values decrease with frequency in a line of sight environment. However, the root mean square delay spread in a non-line of sight environment and the coherence bandwidth values in both line of sight and non-line of sight environments do not show significant changes.

An outdoor wideband channel sounding at $2.4 \mathrm{GHz}$ is described in [13]. The distance between the transmitter and the receiver varied from 50 to $150 \mathrm{~m}$. The distancepower gradient is 2.532, path loss (with $9 \mathrm{~dB}$ standard deviation), small-scale or multipath fading (with $5 \mathrm{~dB}$ standard deviation) are reported. The maximum observed multipath fade is $28 \mathrm{~dB}$.

Another campaign was conducted in Seoul [14]. The measurements were done using a wideband channel sounder at $3.7 \mathrm{GHz}$ with a $100 \mathrm{MHz}$ bandwidth. Both line of sight and non-line of sight environments are investigated. The output was presented as a spatial correlation coefficient of low-height links in an urban environment.

A wideband propagation channel at 2.45 and $5.2 \mathrm{GHz}$ was presented in [15]. Channel characteristics as power delay profile, the mean delay, and the delay spread were studied. It was mentioned that the parameters are frequency-independent, whereas the higher frequency signal shows considerably larger path loss than the lower one. Both the correlator-based and recursive Bayesian filter-based ranging estimators were evaluated; both of them provide better performances at $2.45 \mathrm{GHz}$ compared with $5.2 \mathrm{GHz}$. The performance difference increases with decreasing the received power.

Urban macro environment was investigated in [16]. Wideband multiple-input multiple-output measurements around $800 \mathrm{MHz}$ with $50 \mathrm{MHz}$ bandwidth were presented. The antennas with $360^{\circ}$ of azimuth and $90^{\circ}$ of elevation were used for the transmitter and the receiver. The output report contains path loss (path loss exponent $n=$ 3 ), shadow fading (with 8.4 dB standard deviation), delay spread (with $123 \mathrm{~ns}$ mean value and $73.2 \mathrm{~ns}$ standard deviation), angular spread (with $30.8^{\circ}$ mean value and $12.5^{\circ}$ standard deviation for angular spread of departure and $66.9^{\circ}$ mean value and $15.1^{\circ}$ standard deviation for angular spread of arrival), and Ricean K-factor (with $5 \mathrm{~dB}$ mean value and $6.7 \mathrm{~dB}$ standard deviation). 
Measurement campaign [17] at the center frequency of $2.35 \mathrm{GHz}$ with $50 \mathrm{MHz}$ bandwidth was conducted in order to evaluate the performance in an outdoor propagation environment. Signal to noise ratio, spatial diversity, and capacity of different transmission schemes (direct transmission, amplify and forward, and decode and forward relaying) were investigated. Both line of sight and non-line of sight scenarios were involved. The results were depicted in terms of signal to noise ratio, spatial diversity, and capacity. Both amplify and forward, and decode and forward schemes improve the Signal to Noise Ratio, whereas direct transmission improves the capacity in small distances of a line of sight environment. However, by increasing transmitter-receiver distance, the capacity provided by the decode and forward exceeds that provided by the direct transmission. The spatial diversity was also significantly improved by applying the decode and forward scheme. Most of the abovementioned papers depict indoor channels or even outdoor channels but only up to $2 \mathrm{~km}$ and with only vertical co-polarization. Therefore, we filled these gaps by considering both line of sight and macro non-line of sight scenarios over $1.3 \mathrm{GHz}$ and $5.8 \mathrm{GHz}$ frequencies with longer distances $2.089 \mathrm{~km}$, $4.11 \mathrm{~km}$, and $5.429 \mathrm{~km}$ and both vertical and horizontal co-polarization dependence of multipath propagation channel measurements.

\subsection{Contribution of the paper}

We analyzed the channel frequency response variation, the path loss, the root mean square delay spread, and the coherence bandwidth with all above mentioned scenarios. Our achieved results expand the achieved results in [18] which were performed in an indoor environment. The main contributions of this paper are described in the following few points:

- Test the ability of deploying a device to device communication underlay 5 th generation network in a wideband long-range channel for both ultra high frequency and super high frequency bands as a part of 5th generation new radio frequency bands allocation [19]

- For a microcell line of sight environment (with $315 \mathrm{~m}$ distance), we provided the channel frequency response variation, the path loss, and the root mean square delay spread distribution in the case of vertical and horizontal polarizations for both $1.3 \mathrm{GHz}$ and $5.8 \mathrm{GHz}$ center frequencies.

- For macrocell non-line of sight environments (with $2.089,4.11$, and $5.429 \mathrm{~km}$ distances), in additional of all previous mentioned parameters, distance dependence of the path loss and the root mean square delay spread are analyzed. The root mean square delay spread dependence of the coherence bandwidth is also investigated.
This paper is organized as follows. The "Materials and methods" section describes the channel measurement campaign of outdoor long-range environments and the sounder systems for ultra high frequency and super high frequency bands. The data processing procedure and channel characteristics calculation are also depicted. In the "Results and discussion" section, the channel measurement results are captured for line of sight and nonline of sight outdoor environments with different polarization combinations. Based on channel measurement results, the root mean square delay spread, the path loss, the channel frequency response variation, and the coherence bandwidth are analyzed. The last section concludes the paper.

\section{Materials and methods}

\subsection{Measurement environments and setup}

Our measurements were conducted in the South Moravian region, Czech Republic. Two types of setups: microcell and macrocell were considered.

In the microcell setup, the TX1 was placed on a small hill near the Faculty of Electrical Engineering and Communication building, Brno University of Technology (BUT) and mounted on a mast of $10 \mathrm{~m}$ height, whereas the receiver was allocated on the rooftop of the building (19-m height). The distance between the transmitter and the receiver for route R1 is $315 \mathrm{~m}$. Both the transmitter and the receiver are surrounded by a rich scattering environment which consists of buildings, parked cars, moving cars, and people. However, because of the highly mounted antennas above the ground, line of sight measurements were realized.

In the macrocell setup, three different non-line of sight routes (R2, R3, R4) were tested. On the first two routes $(R 2, R 3)$, the receiver was placed on the rooftop of the Faculty of Electrical Engineering and Communication building. The transmitter was allocated $2.089 \mathrm{~km}$ from the receiver (8-m height) for the $\mathrm{R} 2$ route, and $5.429 \mathrm{~km}$ from the receiver ( $3 \mathrm{~m}$ height) in the case of the $\mathrm{R} 3$ route. For the fourth route, $\mathrm{R} 4$, both the transmitter and the receiver were allocated in a rural area where the transmitter was surrounded with different building heights (up to 12-m height) and placed on the rooftop of the Racom company building (12-m height) mounted on a mast of 5-m height. The receiver was mounted on a mast of $19-\mathrm{m}$ height in a pure rural area. Examples of transmitting antennas in the case of R2 and R3 and receiving antenna with their surrounding environments are presented on the left-hand side, the right-hand side, and the center of Fig. 1, respectively. The mast of the received antenna of the fourth route, R4, is captured on the right-hand side of Fig. 2.

In order to investigate line of sight and non-line of sight radio channel characteristics, two different channel sounder systems for $1.3 \mathrm{GHz}$ and $5.8 \mathrm{GHz}$ were 

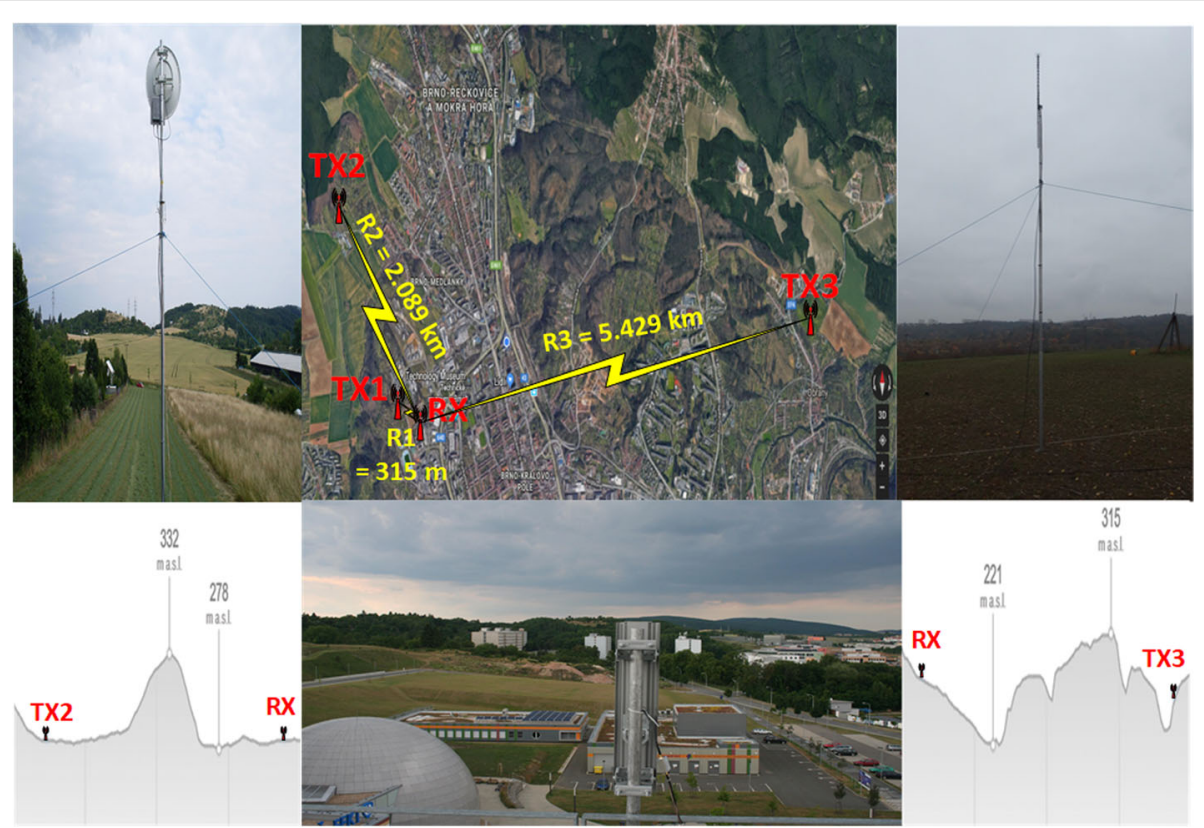

Fig. 1 Measurement locations for R1, R2, and R3 routes. Line of sight route R1 = $315 \mathrm{~m}$ and non-line of sight route $\mathrm{R} 2=2.089 \mathrm{~km}$ with super high frequency band TX2 position on the left-hand side, R3 $=5.429 \mathrm{~km}$ with ultra high frequency band TX3 position on the right-hand side and the position of the sector receiver antenna used for the super high frequency signal of TX2-RX measurements. Map source: Google.com, Mapy.cz

implemented. These sounders together with MATLAB and LabVIEW programs were used for channel evaluation up to $120 \mathrm{MHz}$ and $600 \mathrm{MHz}$ bandwidths for both 1.3 and $5.8 \mathrm{GHz}$, respectively.

The basis of the transmitting station is a programmable radio frequency generator (R\&S SMU200A vector signal generator). The generated signal was filtered using a band pass filter, amplified by a power amplifier, and directed to the directional antenna transmitter using a circular connector. The amplifier module for the ultra high frequency band (MD220L-1296-48V) was modified to be used as a linear amplifier class A. However, the super high frequency band transmitter uses Hittite HMC408LP3 and DG0VE PA6-1-8W amplifying modules. The generated

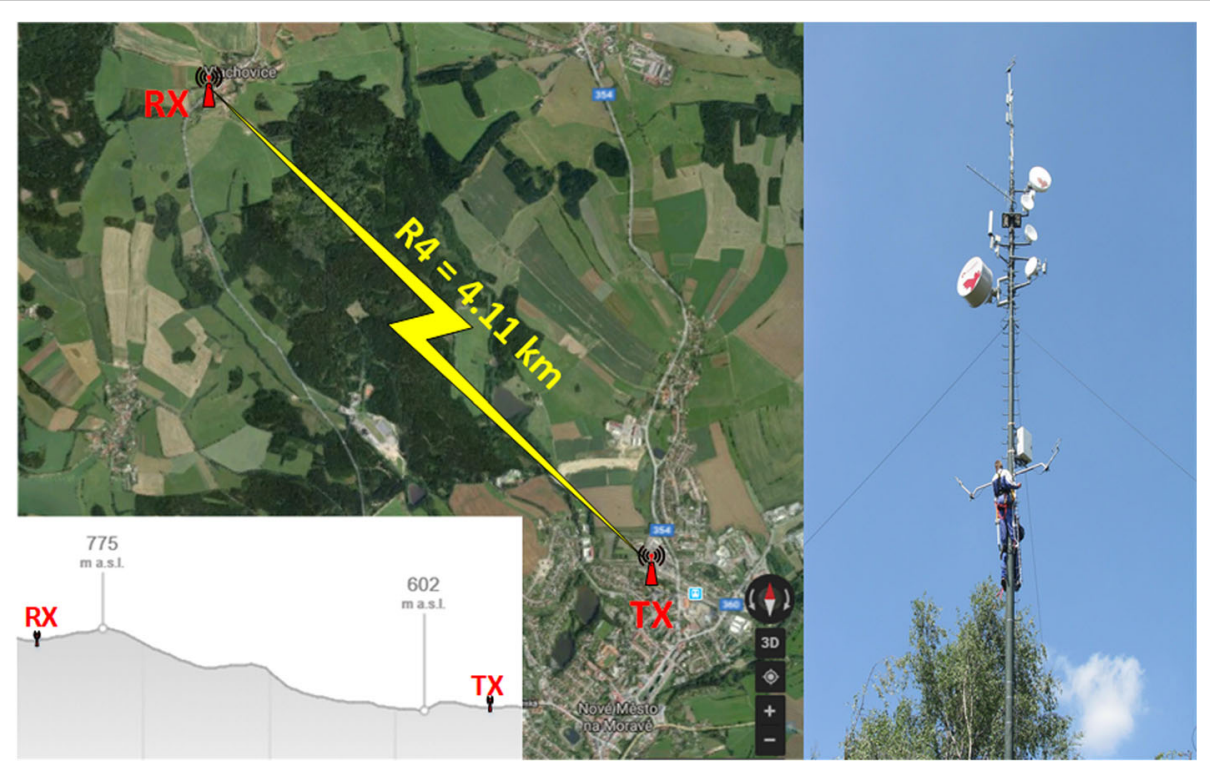

Fig. 2 Measurement location for R4 route. Non-line of sight R4 $=4.11 \mathrm{~km}$ route and the position of the receiver antenna on the middle of the mast. Map source: Google.com, Mapy.cz 
ultra high frequency $(1.3 \mathrm{GHz})$ signal was transmitted using 35-element Yagi Tonna antenna 20365 with $20 \mathrm{dBi}$ of gain. The super high frequency $(5.8 \mathrm{GHz})$ signal was transmitted by a parabolic RD-5G30-LW RocketDish with a gain equaling $30 \mathrm{dBi}$.

The receiver consists of a directional antenna, low-noise amplifier, and signal analyzer (National Instruments PXIe5665) with three basic modules: PXIe-5653 RF synthesizer, PXIe-5605 downconverter, and PXIe-5622 $150 \mathrm{MS} / \mathrm{s}$ 16-bit digitizer. The ultra high frequency and super high frequency signals were received by 35 -element Yagi Tonna antenna 20365 (20 dBi gain) and sector antenna AM-V5G$\mathrm{Ti}$ (21 dBi gain), respectively.

The developed software in LabVIEW environment for National Instruments PXIe-5665 was used for recording and processing the raw data received by the channel sounder. This software is able to record up to $600 \mathrm{MHz}$ of bandwidth via stepped re-tuning by $50 \mathrm{MHz}$ blocks with the ability to be synchronized with the transmitted signal. In order to save the achieved data with $50 \mathrm{MHz}$ instance bandwidth and 16-bits precision, a redundant array of inexpensive disks with capacity of 12 TB and 16bit dynamic range was used. MATLAB was also used for final data processing. Our in-house developed channel sounder operates with frequency modulated continuous wave, i.e., as a sounding sequence; we utilize frequency modulation chirps with a maximal measurement speed of $40 \mathrm{MHz} / \mathrm{ms}$. Figure 3 depicts the schematics of the sounder for ultra high frequency (white blocks) and super high frequency (gray blocks) bands, whereas the characteristics ( $\mathrm{E}$ and $\mathrm{H}$ planes) of both parabolic and sector antennas for vertical and horizontal co-polarizations are captured in Fig. 4.

\subsection{Data processing}

\subsubsection{Channel response}

The radio channel is commonly characterized by scattering, attenuation, reflection, refraction, and fading [20]. In both the wired and wireless communications, the Additive White Gaussian Noise channel is assumed as a basic channel model. More advanced models including fading effects, e.g. the International Telecommunication Union path loss models like Flat Rayleigh, Pedestrian (Ped), and Vehicular (Veh) [21]. The Flat Rayleigh fading channel has a constant attenuation factor during the subframe time and the whole allocated bandwidth. Other two models define two different test environments: outdoor to indoor pedestrian and vehicular wellestablished channel models used for research purposes in mobile communication systems. The impulse response $h$ of the multipath channel can be calculated according to Eq. (1), where $\beta_{w}, \tau_{w}$, and $\varphi_{w}$ represent the amplitude, arrival time, and phase that characterize the $N_{\mathrm{p}}$ number of individual paths between the transmitter and the receiver [21].

$$
h(t, \tau)=\sum_{w=1}^{N_{\mathrm{p}}} \beta_{w}(t) \cdot \delta\left(t-\tau_{w}(t)\right) e^{-j \varphi_{w}(t)}
$$

The frequency response can be measured directly by collecting the measurements of the $s_{21}$ scattering parameter of a radio channel in the frequency domain. It can also be calculated by applying the Fourier transformation on the time domain measurements expressed in Eq. (1). The result could be given by Eq. (2) [22].

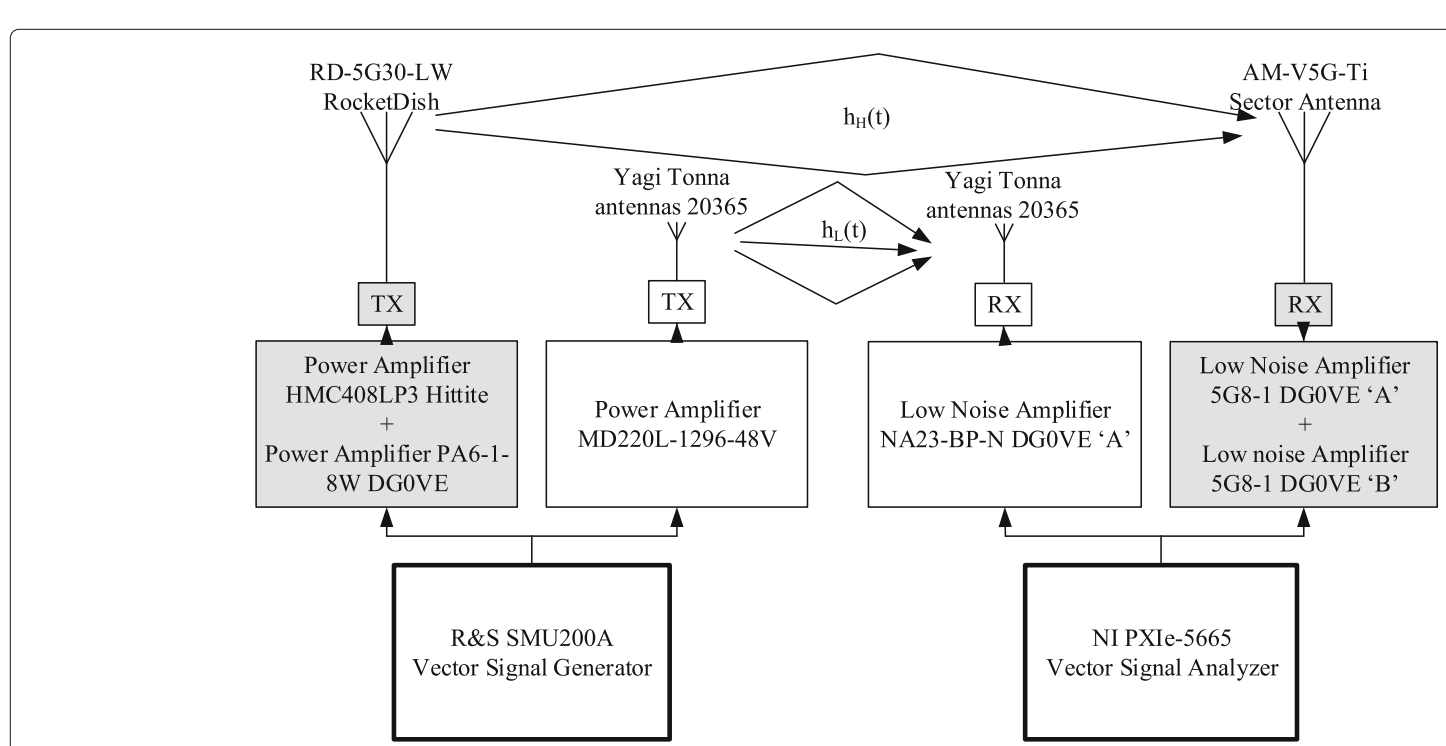

Fig. 3 Channel sounder setup. Channel sounding systems diagram with transmitter and receiver for both ultra high frequency (white colored) and super high frequency (gray colored) bands 

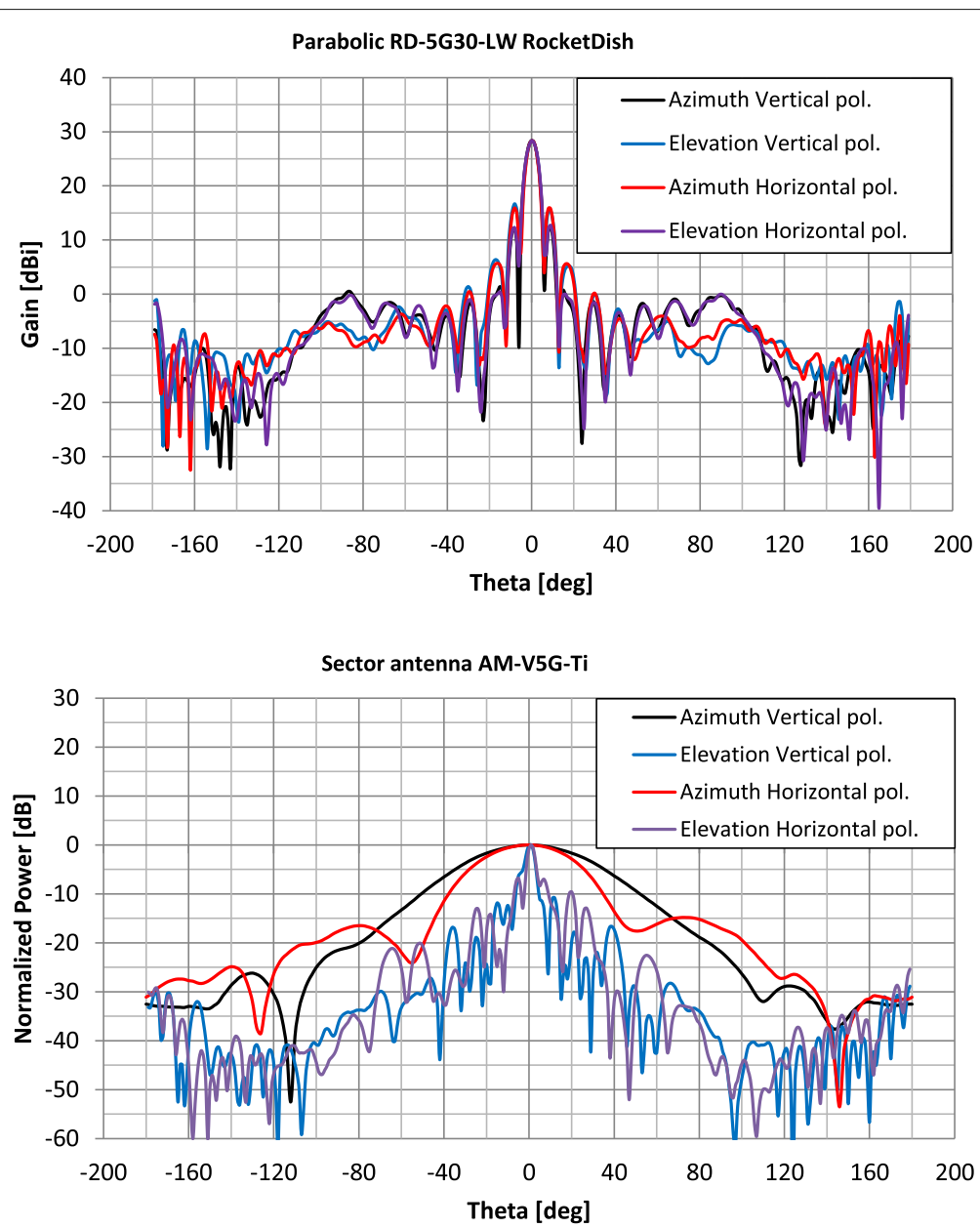

Fig. 4 Parabolic and sector antennas rectangular radiation. Both E and $\mathrm{H}$ planes in the case of vertical and horizontal co-polarizations are captured

$$
\begin{aligned}
H(f, t) & =\int_{-\infty}^{\infty} h(t, \tau) \cdot e^{-j \omega \tau} d \tau \\
& =\sum_{w=1}^{N_{\mathrm{p}}} \beta_{w}(t) e^{-j \varphi_{w}(t)} e^{-j \omega \tau_{w}(t)}
\end{aligned}
$$

In a slowly time-varying channel, the multipath parameters of the channel remain constant during fractions of the coherence time of the channel; so the frequency response can be presented in Eq. (3).

$$
H(f)=H(f, 0)=\sum_{w=1}^{N_{\mathrm{p}}} \beta_{w} e^{-j \varphi_{w}} e^{-j \omega \tau_{w}}
$$

In practice, however, the measurement systems are band-limited. Therefore, the frequency response is defined in Eq. (4).

$$
H(f)=H(f, 0)=W(f) \cdot \sum_{w=1}^{N_{p}} \beta_{w} e^{-j \varphi_{w}} e^{-j \omega \tau_{w}}
$$

where $W(f)$ represents the frequency domain RF filter characteristics in the frequency domain.

\subsubsection{Path loss}

The generalized form of the path loss model can be constructed from path loss offset $P L_{\text {offset }}$, the distance $d$ between transmitter and receiver, the reference distance $d_{0}$, and the random shadowing effect $\chi_{\sigma}$ which is calculated as the deviation of the measured path loss from the linear model [12].

$$
P L(d)=P L_{\text {offset }}+10 n \cdot \log \left(\frac{d}{d_{0}}\right)+\chi_{\sigma}
$$

where $n$ is a path loss exponent and $d_{0}=100 \mathrm{~m}$ for long outdoor distances [20].

\subsubsection{Root mean square delay spread}

The root mean square delay spread is one of the most important parameters for the delay time extent of a multipath radio channel. It is caused by reflected and scattered propagation paths. It can describe different multipath 
fading channels and a guideline to design a wireless transmission system. If $\tau_{w}$ is the channel delay of $w$ th path and $P\left(\tau_{w}\right)$ is its power, then the root mean square delay spread can be formulated in Eq. (6).

$$
\sigma_{\tau}=\sqrt{\overline{\tau^{2}}-\tau_{m}^{2}}
$$

where $\tau_{m}$ is the mean excess delay and it is given by Eq. (7).

$$
\begin{array}{r}
\tau_{m}=\frac{\sum_{w=1}^{N_{p}} P\left(\tau_{w}\right) \tau_{w}}{\sum_{w=1}^{N_{p}} P\left(\tau_{w}\right)} \\
\overline{\tau^{2}}=\frac{\sum_{w=1}^{N_{p}} P\left(\tau_{w}\right) \tau_{w}^{2}}{\sum_{w=1}^{N_{p}} P\left(\tau_{w}\right)}
\end{array}
$$

\subsubsection{Coherence bandwidth}

Coherence bandwidth is a statistical measure of range of frequencies over which the channel can be considered as a flat channel. In other words, coherence bandwidth is the range of frequencies over which two frequency components have a strong potential for amplitude correlation. In the case where the coherence bandwidth is defined as a bandwidth with correlation of 0.5 or above, it can be calculated using the frequency correlation function depicted in Eq. (10) [12].

$$
S(\Delta f) \cong \int_{-\infty}^{\infty} H(f) H^{*}(f+\Delta f) \cdot d f
$$

$H(f)$ is the complex transfer function of the channel, $\Delta f$ is frequency shift and denotes the complex conjugate.

$$
B_{c, 0.5} \approx \min \left(\Delta f: \frac{S(\Delta f)}{S(0)}<0.5\right)
$$

\subsubsection{Channel frequency response variation}

Let us consider that $H_{s}\left(f_{k}\right), k=1,2, \cdots N_{F}, s=$ $1,2, \cdots, N_{T}$ is the wideband channel frequency response at specific time for a specific frequency. Figure 5 depicts a sample $\left(N_{T}=200\right)$ of normalized channel frequency response in $\mathrm{dB}$ where $f_{1}=1.2702 \mathrm{GHz}, f_{2}=1.31 \mathrm{GHz}$, therefore $\Delta f=f_{2}-f_{1}=39.758 \mathrm{MHz}$, and $N_{F}=1554$. The influence of variation and small-scale fading can be removed by averaging consecutive $N_{T}$ channel frequency response characteristics [23] according to Eq. (11).

$$
\overline{\left|H\left(f_{k}\right)\right|^{2}}=\frac{1}{N_{T}} \cdot 10 \cdot \log \left(\sum_{s=1}^{N_{T}}\left|H_{s}\left(f_{k}\right)\right|^{2}\right)
$$

However, as is mentioned in [24], averaging keeps some small-scale fluctuations in the frequency domain; therefore, the median filter is applied. The median filter is a non-linear filter used to discard the noise from the signal. The main idea is to run through the whole signal and calculate the median of each window [25]. Note that in order to get a well-filtered signal, a proper window size of median filter (that keeps the deep fades effect) should be chosen. In our research, the window size will depend primarily on the deep fades where the signal strength can drop for more than $15 \mathrm{~dB}$. Therefore, the window size of 10 is chosen as the average frequency distance of the deep

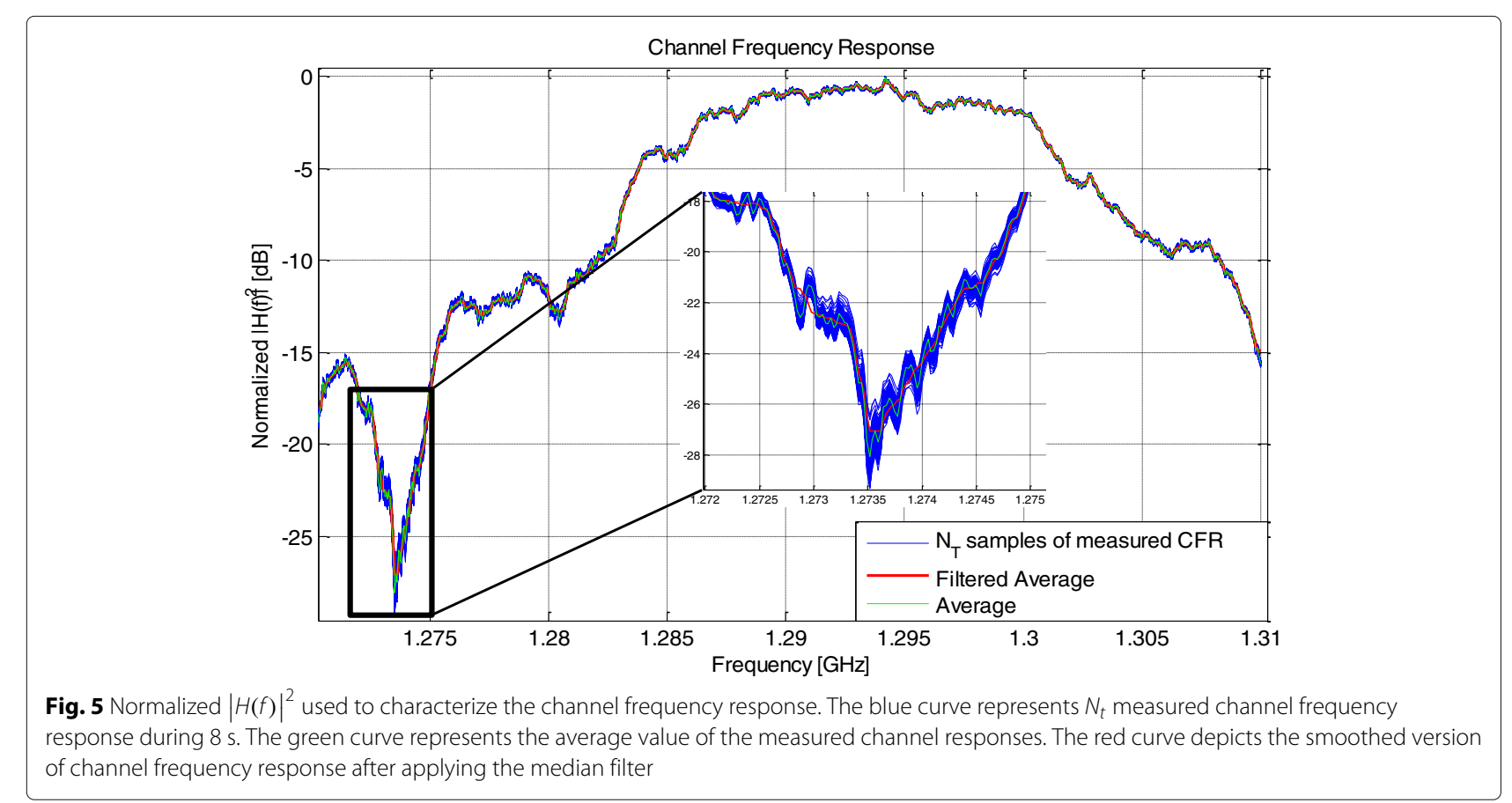


fades. Finally, the channel frequency response variation is obtained through subtracting the filtered average channel frequency response from the measured channel frequency response.

$$
\mathrm{CFRV}=\left|H_{s}\left(f_{k}\right)\right|^{2}-\text { filt }\left(\overline{\left|H\left(f_{k}\right)\right|^{2}}\right)
$$

\section{Results and discussion}

\subsection{Path loss}

Figure 6 presents the cumulative distribution function of the path loss for a line of sight environment. The cumulative probability of path loss values fit well with the normal distribution with $\mu$ mean and $\sigma$ standard deviation parameters.

The path loss values of the R1 line of sight route are in the range of $87.6-88.5 \mathrm{~dB}$ with a mean value $88.1 \mathrm{~dB}$ and standard deviation $0.22 \mathrm{~dB}$ for $1.3 \mathrm{GHz}$ with horizontal copolarization, $88.2-89.9 \mathrm{~dB}$ with a mean value $89.04 \mathrm{~dB}$ and standard deviation $0.33 \mathrm{~dB}$ for $1.3 \mathrm{GHz}$ with vertical copolarization, $114.9-116.1 \mathrm{~dB}$ with a mean value $115.49 \mathrm{~dB}$ and standard deviation $0.23 \mathrm{~dB}$ for $5.8 \mathrm{GHz}$ horizontal co-polarization, and $116.5-117.9 \mathrm{~dB}$ with a mean value $117.1 \mathrm{~dB}$ and standard deviation $0.24 \mathrm{~dB}$ for $5.8 \mathrm{GHz}$ with vertical co-polarization.

The distribution shape is also depicted in Fig. 6 and presented in black, blue, magenta, and cyan colors for ultra high frequency horizontal co-polarization, ultra high frequency vertical co-polarization, super high frequency horizontal co-polarization, and super high frequency vertical co-polarization, respectively. The shape can provide useful information about the density of the calculated path loss. It can be observed that in the case of both 1.3 and 5.8 $\mathrm{GHz}$, the path loss for vertical co-polarization exceeds the path loss of the horizontal one. This small difference (1$2 \mathrm{~dB}$ ) is explained by the effect of suppression which can influence either the vertical or the horizontal polarization. That depends on the distance between transmitter and receiver, their heights, and the type of ground [26, 27]. It is also clear that the path loss increases with frequency as the higher frequencies tend to suffer greater signal absorption and scattering. The same characteristics are observed in $[6,8,20]$ higher frequencies tend to suffer greater signal absorption.

Figure 7 presents the measured path loss for $1.3 \mathrm{GHz}$ sounding system in the case of horizontal and vertical co-polarizations. Black circles represent the measured path loss values for horizontally transmitted and received signals, whereas blue circles represent the measured path loss values for vertically transmitted and received signals. The best line fit have been produced using a MATLAB function with path loss exponents equal to 3.9 and 3.7 for horizontal and vertical polarization cases, respectively. These results are comparable with the results specified in [8], where the path loss exponent value for distances up to $1.4 \mathrm{~km}$ varies between 2.9 and 3.1 for $2 \mathrm{GHz}$

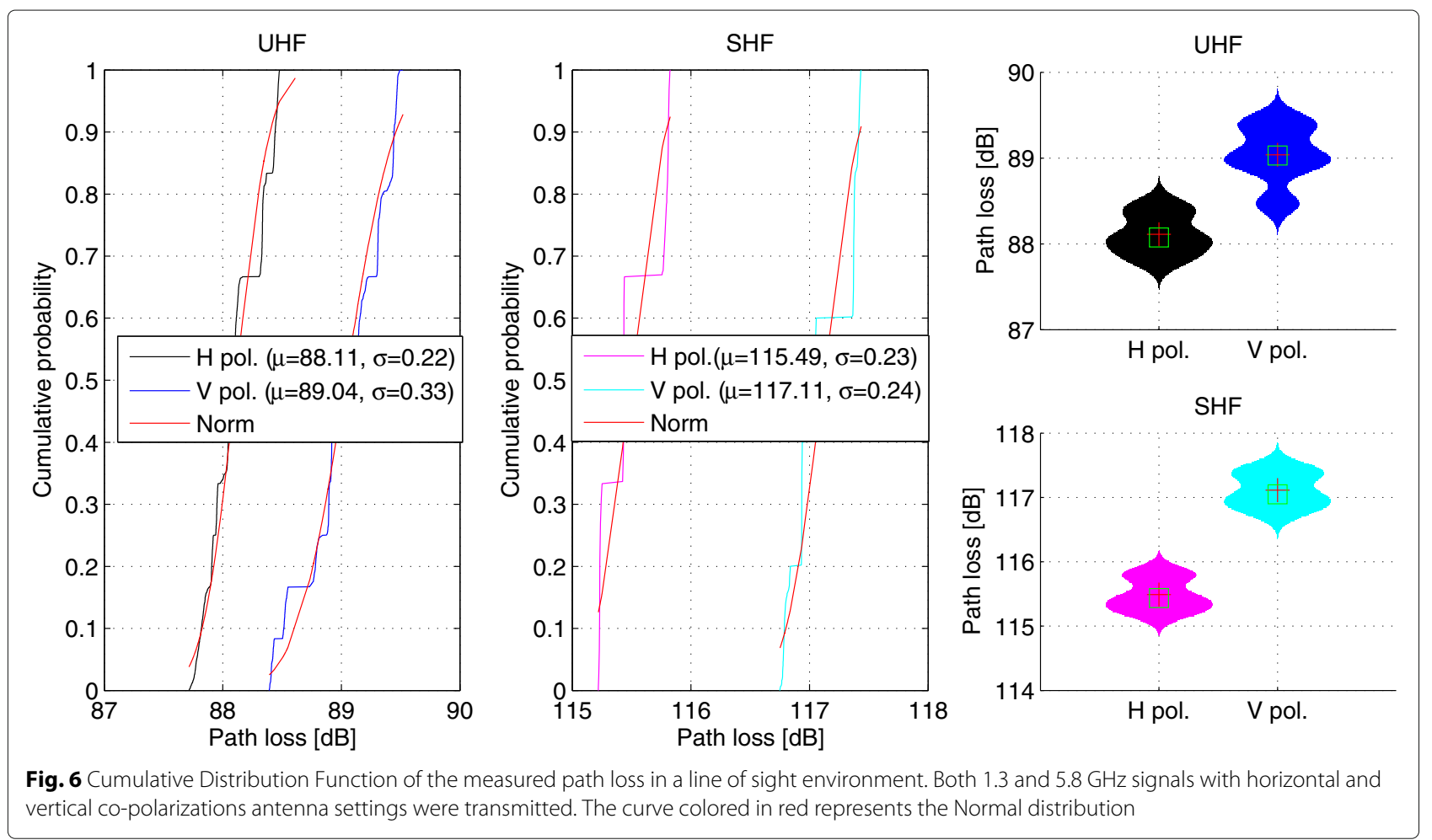




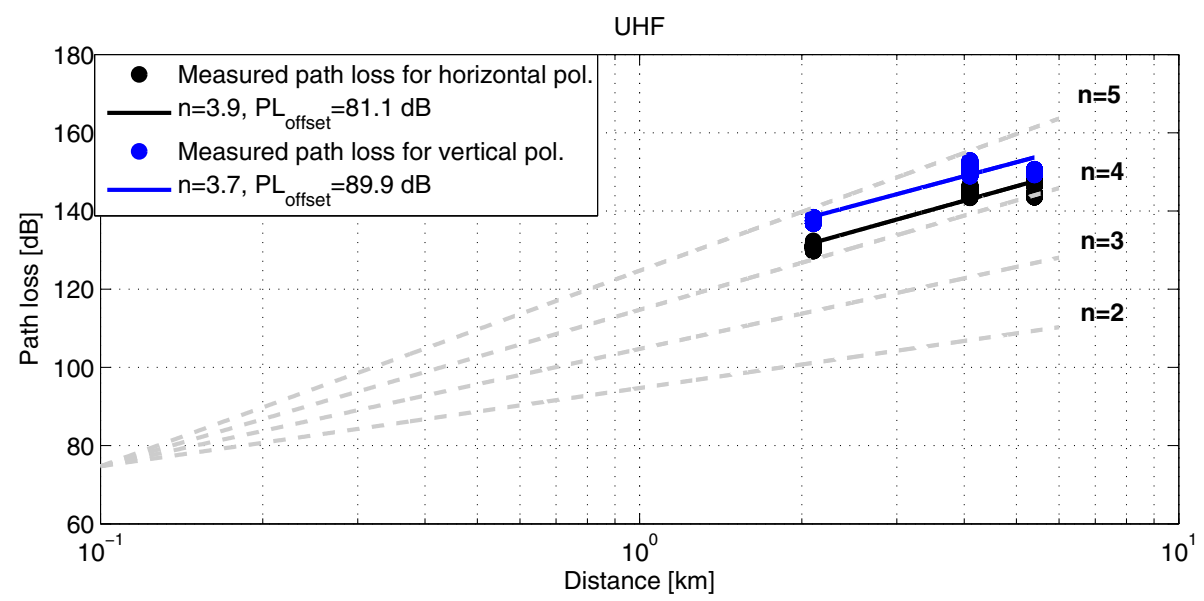

Fig. 7 The measured path loss for $1.3 \mathrm{GHz}$ center frequency of the outdoor non-line of sight environment. The black and blue circles represent the measured non-line of sight path loss values for horizontal and vertical co-polarization, respectively

frequency. The path loss exponent value was also captured in an urban environment [16] for $800 \mathrm{MHz}$ frequency and reached the value of $n=3.3$.

Figure 8 depicts the measured path loss for a $5.8-\mathrm{GHz}$ sounding system in the case of horizontal and vertical co-polarizations, represented by black and blue circles, respectively. The best line fit has been produced using a MATLAB function with path loss exponents $n$ equal to 4.6 and 4.1 for horizontal and vertical polarization cases, respectively. These values can be compared with values achieved in [12], for frequencies $3-6 \mathrm{GHz}$ where the path loss exponents between 3.92 and 4.7 were achieved. According to outdoor measurements presented in [28], the path loss exponent value changes from $n$ equal to 2 to $n$ equal to 4 at the breakpoints distance near $2.85 \mathrm{~km}$.
The dashed gray lines represent the theoretical path loss model in the case of different exponents. Note that the path loss exponent represents the slope of the path loss line, whereas $P L_{\text {offset }}=P L_{F}+P L_{\mathrm{NLOS}}$ where $P L_{F}$ and $P L_{\mathrm{NLOS}}$ are free space path loss and the path loss offset due to non-line of sight environment effects. More information about the path loss values for different line of sight and non-line of sight scenarios are listed in Table 1.

\subsection{Root mean square delay spread}

Figures 9 and 10 display the cumulative probability of the root mean square delay spread for the line of sight environment presented as R1 route and 2.089, 5.429, and $4.11 \mathrm{~km}$ non-line of sight environments presented as R2, $\mathrm{R} 3$, and R4 routes, respectively. The root mean square

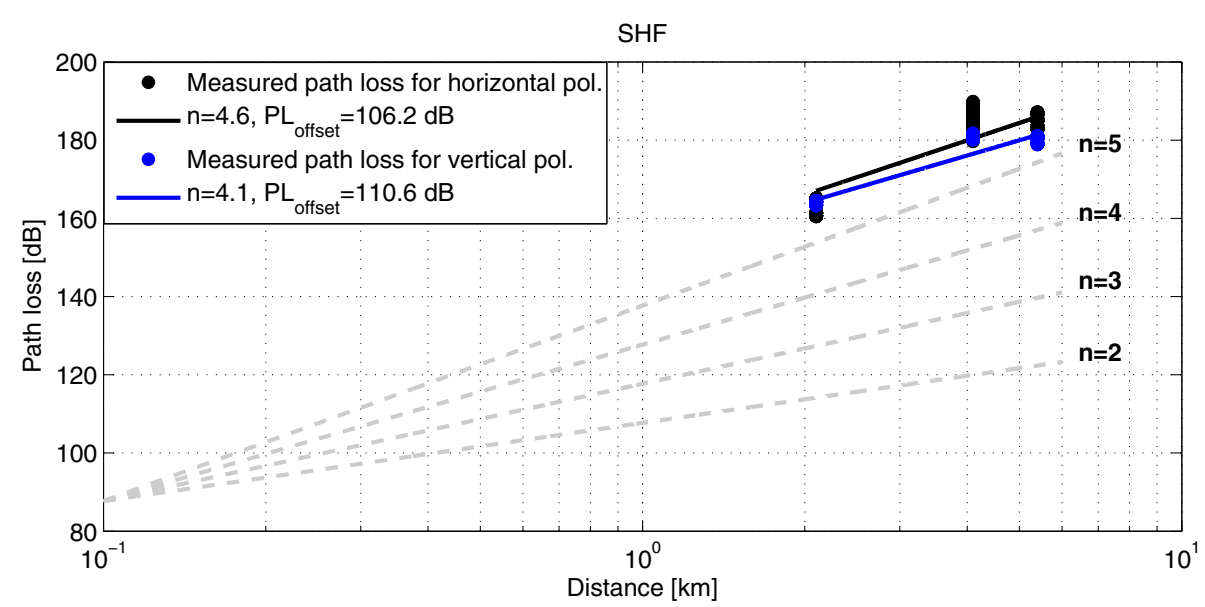

Fig. 8 The measured path loss for $5.8 \mathrm{GHz}$ center frequency of the outdoor non-line of sight environment. The black and blue circles represent the measured non-line of sight path loss values for horizontal and vertical co-polarizations, respectively 
Table 1 The path loss mean, standard deviation, minimum, and maximum values for different frequencies and co-polarizations in both line of sight and non-line of sight environments

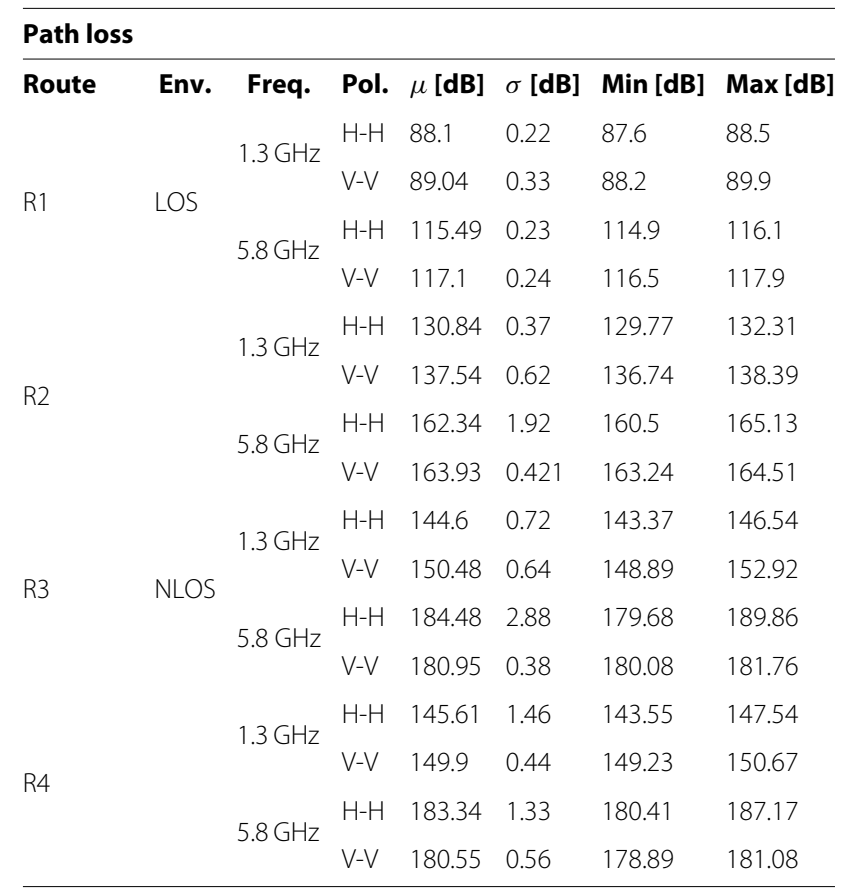

delay spread values for all routes and frequencies fit well with the normal distribution with $\mu$ mean and $\sigma$ standard deviation parameters.

It can be distinguished that the $\mathrm{R} 1$ line of sight route offers smaller root mean square delay spread compared with all plotted root mean square values for non-line of sight environments in both 1.3 and $5.8 \mathrm{GHz}$. This behavior is expected. On the one hand, it can be due to a very strong line of sight component compared with the reflected or scattered path, leading to lower root mean square delay spread. On the other hand, in the case of a non-line of sight environment, the transmitted signal is blocked or severely attenuated causing multipath to arrive at the receiver over a large propagation time interval. Similar characteristics are observed in [12, 29].

The wideband root mean square delay spread for the $\mathrm{R} 1$ route is in the range of $15.11-18.42 \mathrm{~ns}$ for $1.3 \mathrm{GHz}$ with horizontal co-polarization, $23.18-32.58 \mathrm{~ns}$ for $1.3 \mathrm{GHz}$ with vertical co-polarization, $11.53-12.21 \mathrm{~ns}$ for $5.8 \mathrm{GHz}$ horizontal co-polarization, and 15.82 $16.62 \mathrm{~ns}$ for $5.8 \mathrm{GHz}$ with vertical co-polarization.

It can be seen from Fig. 9 that in the case of the first route R1 line of sight, the higher frequency provides smaller mean root mean square delay spread in both horizontal and vertical polarization settings. This behavior was also mentioned in [12, 30]. It is also clear that in the case of both ultra high frequency and super high frequency frequencies, the vertical co-polarization shows higher mean root mean square delay than horizontal copolarization.

For the R2 route, the root mean square delay spread is in the range of $52.99-99.70 \mathrm{~ns}$ for $1.3 \mathrm{GHz}$ with horizontal co-polarization, $77.44-104.82 \mathrm{~ns}$ for $1.3 \mathrm{GHz}$ with vertical co-polarization, $46.43-60.67 \mathrm{~ns}$ for $5.8 \mathrm{GHz}$ horizontal copolarization, and $72.15-87.63 \mathrm{~ns}$ for $5.8 \mathrm{GHz}$ with vertical co-polarization.
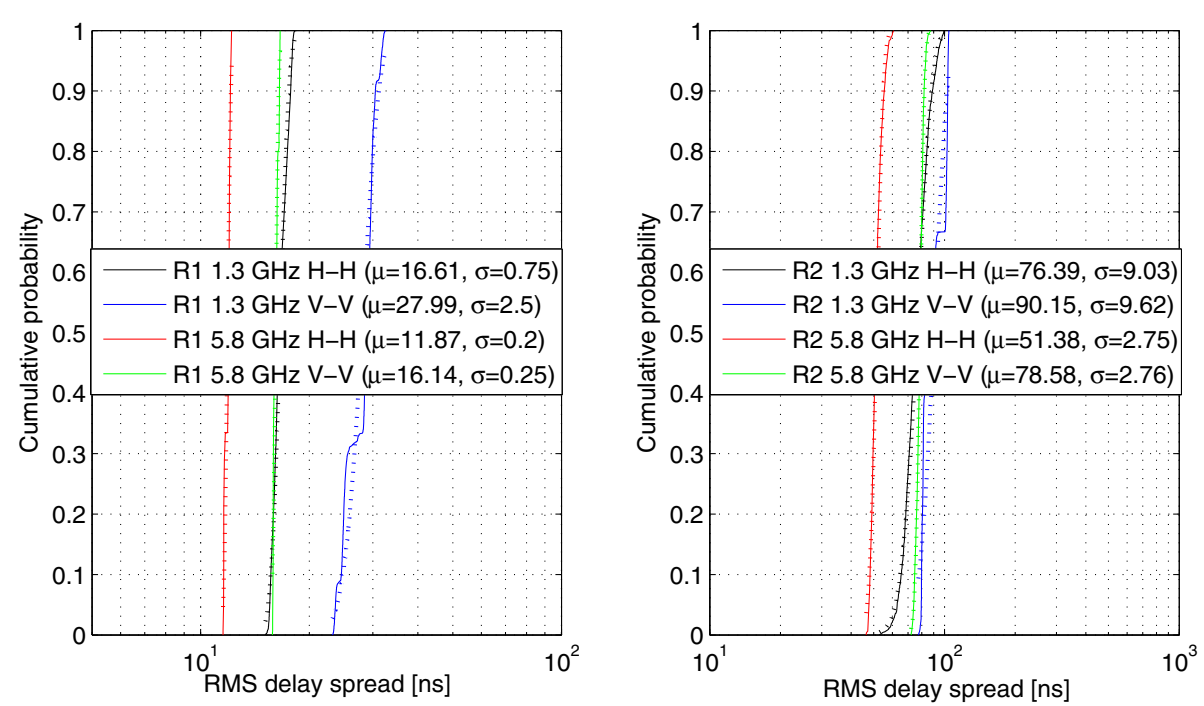

Fig. 9 R1 and R2 root mean square delay spread. Cumulative Distribution Function of the root mean square delay spread in [ns] for different frequencies and both horizontal and vertical co-polarizations of the first and second measurement routes (R1 and R2) in line of sight and non-line of sight scenarios, respectively. The colored dotted lines represent the Normal distribution of the corresponding frequency and polarization combinations 

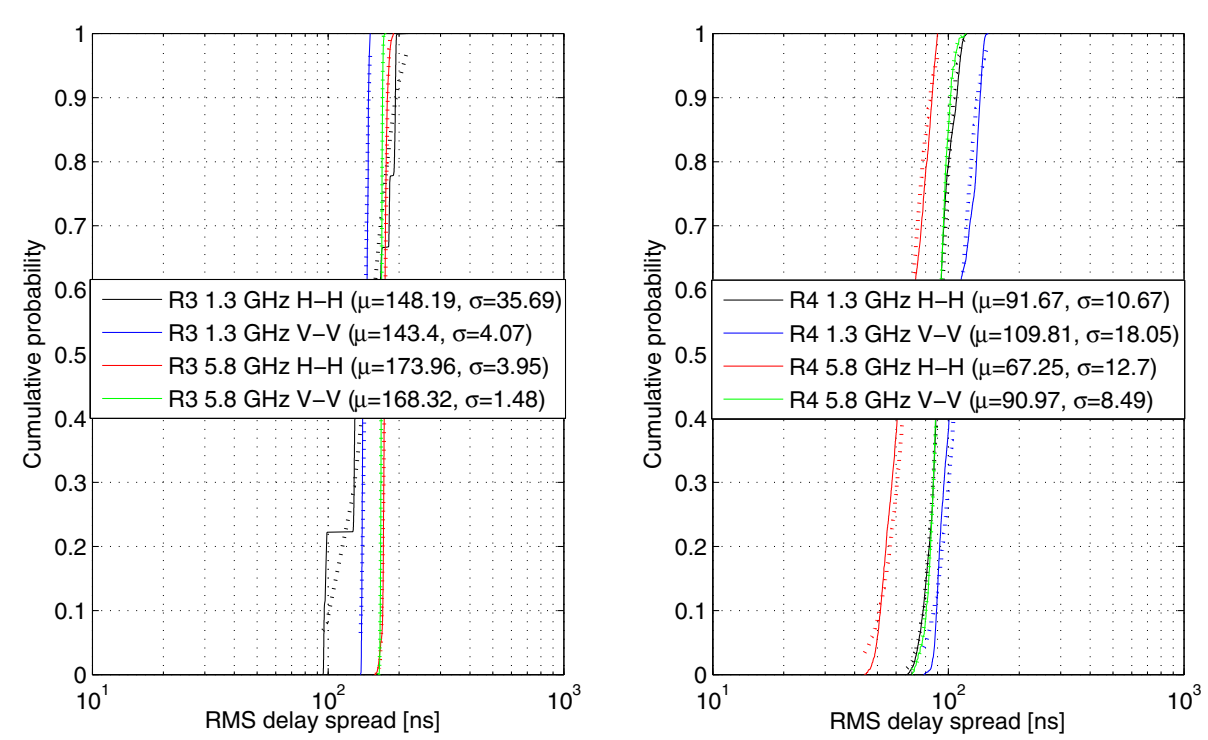

Fig. 10 R3 and R4 root mean square delay spread. Cumulative distribution function of root mean square delay spread in [ns] for different frequencies and both horizontal and vertical co-polarizations captured for the third and fourth measurement routes (R3 and R4). The colored dotted lines represent the normal distribution of the corresponding frequency and polarization combinations

In the case of the $\mathrm{R} 3$ route, the root mean square delay spread is in the range of $95.21-215.39 \mathrm{~ns}$ for $1.3 \mathrm{GHz}$ with horizontal co-polarization, $137.24-151.27 \mathrm{~ns}$ for $1.3 \mathrm{GHz}$ with vertical co-polarization, $157.7-189.54 \mathrm{~ns}$ for $5.8 \mathrm{GHz}$ horizontal co-polarization, and $163.33-176.58 \mathrm{~ns}$ for $5.8 \mathrm{GHz}$ with vertical co-polarization. For the R4 route, it is in the range of $66.85-119.73 \mathrm{~ns}$ for $1.3 \mathrm{GHz}$ with horizontal co-polarization, $78.73-147.76 \mathrm{~ns}$ for $1.3 \mathrm{GHz}$ with vertical co-polarization, $44.12-89.91 \mathrm{~ns}$ for $5.8 \mathrm{GHz}$ horizontal co-polarization, and 70.44-119.11 ns for $5.8 \mathrm{GHz}$ with vertical co-polarization. It can be observed that the horizontal co-polarization shows smaller mean root mean square delay spread than the vertical co-polarization for 1.3 and $5.8 \mathrm{GHz}$. However, the third route, R3, shows different characteristics. This can be due to the building's metal roof between the transmitter and the receiver, that cause depolarization. Table 2 combines all needed information about the root mean square delay spread values.

The effect of transmitter-receiver distance on the mean values of root mean square delay spread is also investigated. The mean root mean square delay spread values increase with the increasing distance between the transmitter and the receiver. A similar trend is observed in [31]. The relation between the mean root mean square delay spread and the distance can be fitted with linear mode $\sigma_{\tau \mathrm{UHF}, H}=20 d+27$ for ultra high frequency with horizontal co-polarization, $\sigma_{\tau \mathrm{UHF}, V}=15 d+55$ for ultra high frequency with vertical co-polarization, $\sigma_{\tau \text { SHF }, H}=34 d-33$ for super high frequency with horizontal co-polarization, and $\sigma_{\tau \mathrm{SHF}, V}=25 d+15$ for super high frequency with vertical co-polarization, where $d$ is the distance between the transmitter and the receiver in kilometers. This characteristic is comparable with results published in [32, 33].

From these functions, it can be noticed that the line slope of the root mean square delay spread of super high

Table 2 The mean, standard deviation, minimum, and maximum values of root mean square delay spread for different frequencies and co-polarizations in both line of sight and non-line of sight environments

\begin{tabular}{|c|c|c|c|c|c|c|c|}
\hline \multicolumn{8}{|c|}{ RMS delay } \\
\hline Route & Env. & Freq. & Pol. & $\mu$ [ns] & $\sigma$ [ns] & Min [ns] & $\operatorname{Max}[n s]$ \\
\hline \multirow{4}{*}{ R1 } & \multirow{4}{*}{ LOS } & \multirow{2}{*}{$1.3 \mathrm{GHz}$} & $\mathrm{H}-\mathrm{H}$ & 16.61 & 0.75 & 15.11 & 18.42 \\
\hline & & & $V-V$ & 27.99 & 2.50 & 23.18 & 32.58 \\
\hline & & \multirow{2}{*}{$5.8 \mathrm{GHz}$} & $\mathrm{H}-\mathrm{H}$ & 11.87 & 0.20 & 11.53 & 12.21 \\
\hline & & & $V-V$ & 16.14 & 0.25 & 15.82 & 16.62 \\
\hline \multirow{4}{*}{ R2 } & \multirow{12}{*}{ NLOS } & \multirow{2}{*}{$1.3 \mathrm{GHz}$} & $\mathrm{H}-\mathrm{H}$ & 76.39 & 9.03 & 52.99 & 99.70 \\
\hline & & & $V-V$ & 90.15 & 9.62 & 77.44 & 104.82 \\
\hline & & \multirow{2}{*}{$5.8 \mathrm{GHz}$} & $\mathrm{H}-\mathrm{H}$ & 51.38 & 2.75 & 46.43 & 60.67 \\
\hline & & & $V-V$ & 78.58 & 2.76 & 72.15 & 87.63 \\
\hline \multirow{4}{*}{ R3 } & & \multirow{2}{*}{$1.3 \mathrm{GHz}$} & $\mathrm{H}-\mathrm{H}$ & 148.19 & 35.69 & 95.21 & 215.39 \\
\hline & & & $V-V$ & 143.40 & 4.07 & 137.24 & 151.27 \\
\hline & & \multirow{2}{*}{$5.8 \mathrm{GHz}$} & $\mathrm{H}-\mathrm{H}$ & 173.96 & 3.95 & 157.70 & 189.54 \\
\hline & & & $V-V$ & 168.32 & 1.48 & 163.33 & 176.58 \\
\hline \multirow{4}{*}{ R4 } & & \multirow{2}{*}{$1.3 \mathrm{GHz}$} & $\mathrm{H}-\mathrm{H}$ & 91.67 & 10.67 & 66.85 & 119.73 \\
\hline & & & $V-V$ & 109.81 & 18.05 & 78.73 & 147.76 \\
\hline & & \multirow{2}{*}{$5.8 \mathrm{GHz}$} & $\mathrm{H}-\mathrm{H}$ & 67.25 & 12.70 & 44.12 & 89.91 \\
\hline & & & $V-V$ & 90.97 & 8.49 & 70.44 & 119.11 \\
\hline
\end{tabular}


frequency frequencies greater than the line slope of root mean square delay spread of ultra high frequency frequencies. Therefore, the mean root mean square delay spread for super high frequency frequencies more extremely increases compared with the mean root mean square delay spread of ultra high frequency frequencies. The same characteristics were captured in the case of comparing horizontal and vertical co-polarization where the horizontally polarized signal is more sensitive to distance changes. All above mentioned properties are depicted in Fig. 11.

\subsection{Coherence bandwidth}

Figure 12 depicts the root mean square delay spread dependency of the coherence bandwidth in $1.3 \mathrm{GHz}$ nonline of sight environments where the coherence bandwidth in $\mathrm{MHz}$ and the root mean square delay spread in ns. This relation is fitted with an exponential model $B_{c, 0.5}=18.34 \cdot e^{-0.002 \sigma_{\tau}}$.

Figure 13 depicts root mean square delay spread dependency of the coherence bandwidth in $5.8 \mathrm{GHz}$ non-line of sight environments where the coherence bandwidth is in megahertz and the root mean square delay spread is in nanoseconds. The measurements represented by blue circles, which were achieved from R2, R3, R4 route measurements, fit with the exponential model $B_{c, 0.5}=121.5$. $e^{-0.014 \sigma_{\tau}}$. A similar relation was observed in $[34,35]$.

\subsection{Channel frequency response variation}

Figure 14 depicts the cumulative probability of the measured wideband channel frequency response variation for different routes ( $\mathrm{R} 1, \mathrm{R} 2, \mathrm{R} 3, \mathrm{R} 4$ ) of $1.3 \mathrm{GHz}$ center frequency and horizontal and vertical co-polarizations. It can be observed that the channel frequency response variation values fit well with the Normal distribution which is plotted as a dotted curve colored according to a particular route. The channel frequency response variations have mean values of $0.044,0.25,0.288$, and $0.182 \mathrm{~dB}$ and standard deviation of $0.036,0.291,0.403$, and $0.196 \mathrm{~dB}$ in the case of horizontal co-polarization, whereas the mean values of $0.06,0.127,0.377$, and $0.295 \mathrm{~dB}$ and standard deviation of $0.078,0.172,0.537$, and $0.289 \mathrm{~dB}$ in the case of vertical co-polarization. It can be noticed that the channel frequency response variations have the smallest mean and standard deviation values in the case of the $\mathrm{R} 1$ route which corresponds to the line of sight scenario.

Figure 15 presents the cumulative probability of the measured wideband channel frequency response variation for different routes (R1, R2, R3, R4) of $5.8 \mathrm{GHz}$ center frequency and horizontal and vertical co-polarizations. It can be seen that the channel frequency response variation values also fit well with the Normal distribution which is plotted as a dotted curve colored according to a particular route. The channel frequency response variations have mean values of $0.041,3.083,1.246$, and $2.296 \mathrm{~dB}$ and standard deviation of $0.119,2.902,1.686$, and $2.3 \mathrm{~dB}$ in the case of horizontal co-polarization, whereas the mean values of $0.044,3.48,1.621$, and $1.491 \mathrm{~dB}$ and standard deviation of $0.119,2.952,1.621$, and $1.478 \mathrm{~dB}$ in the case of vertical co-polarization. The same feature of lowest mean and standard deviation values appears for the first route R1 which represents the line of sight scenario.

It is also clear from Figs. 14 and 15 that the channel frequency response variations increase with frequency. This merit becomes more visible in the case of the non-line of sight scenario. The second route R2 shows the highest channel frequency response variation due to higher frequency signals which tend to scatter more than the lower ones. These scatter objects can be moving people and cars.

\section{Conclusion}

In this paper, a device to device outdoor long-range communication channel was utilized for a measurement campaign. Both ultra high frequency and super high frequency channels were sounded using Yagi Tonna antennas as a
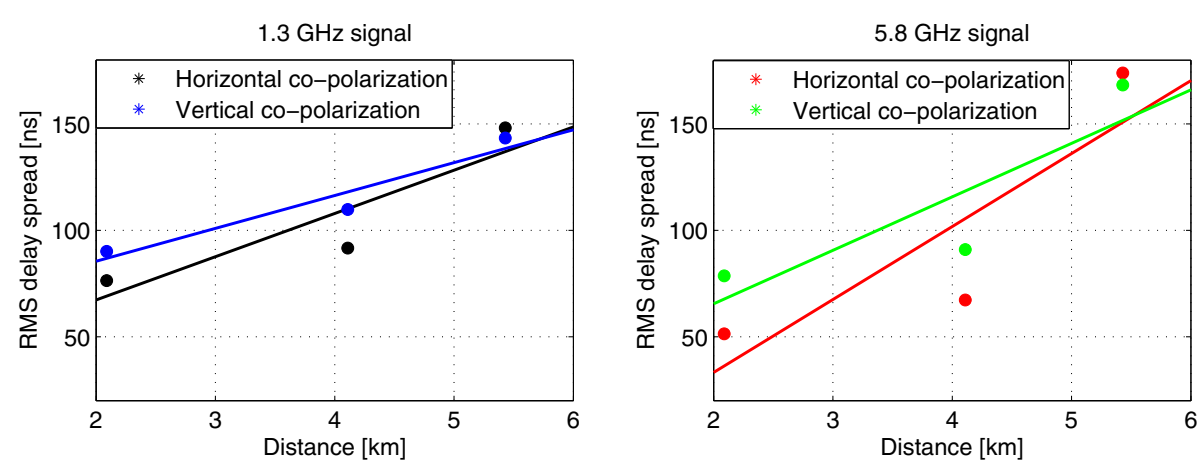

Fig. 11 Root mean square delay spread versus distance. The mean values of root mean square delay spread as a function of transmitter-receiver distance for different frequencies and co-polarizations in a non-line of sight environment 


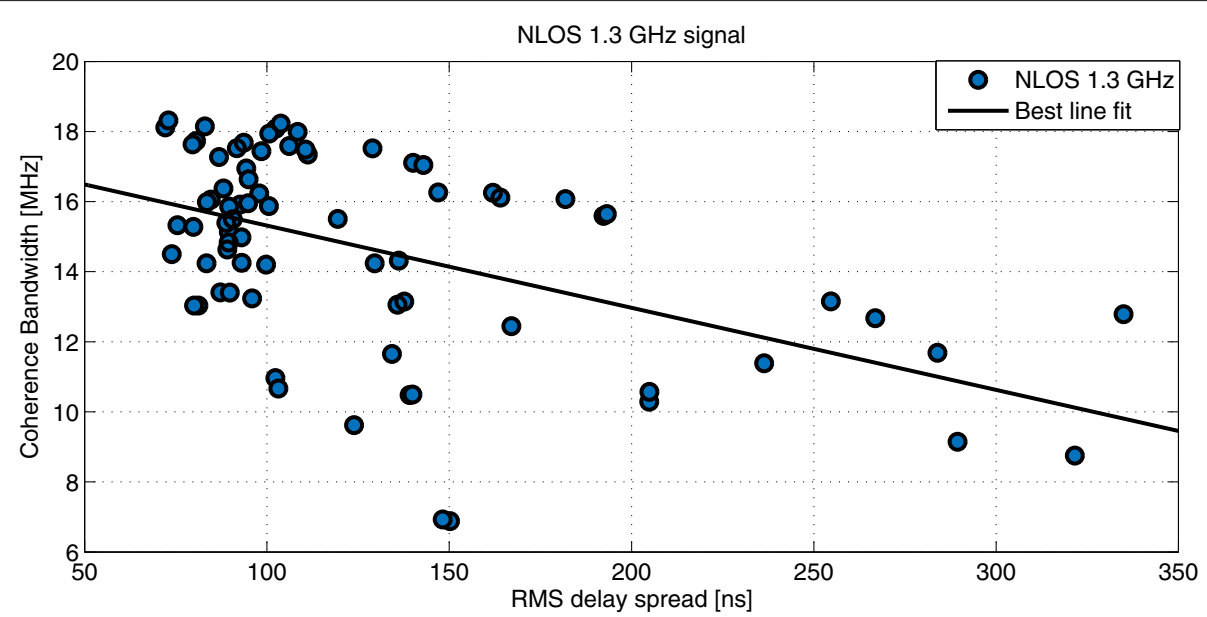

Fig. 12 Coherence bandwidth versus root mean square delay spread for $1.3 \mathrm{GHz}$. Scatter plot of the coherence bandwidth $B_{c, 0.5}$ against the root mean square delay spread in the case of a transmitted signal with $1.3 \mathrm{GHz}$ center frequency in non-line of sight environments

transmitter and a transmitter at $1.3 \mathrm{GHz}$ and a parabolic RD-5G30-LW RocketDish antenna transmitter and AMV5G-Ti sector antenna receiver at $5.8 \mathrm{GHz}$. The vertical and the horizontal co-polarizations were presented in both line of sight and non-line of sight scenarios. As output, channel characteristics such as root mean square delay spread, path loss, coherence bandwidth, and channel frequency response variation were extracted.

In the case of microcell LOS environment (with 315-m distance), the mean path loss value for vertical copolarization exceeds the mean path loss value of the horizontal one by $0.93 \mathrm{~dB}$ and $1.62 \mathrm{~dB}$ in the case of ultra high frequency and super high frequency bands, respectively. It was observed that the path loss increases with frequency about 27 and $28 \mathrm{~dB}$ in the case of horizontal and vertical co-polarizations, respectively. Moreover, the higher frequency provides smaller mean root mean square delay spread in both horizontal and vertical polarizations settings. It was also mentioned that the vertical copolarization shows higher mean root mean square delay than horizontal co-polarization. Finally the channel frequency response variations were negligible in the case of ultra high frequency and super high frequency channel sounding with horizontal and vertical polarization cases.

In the case of macrocell non-line of sight environments (with $2.089,4.11$, and $5.429 \mathrm{~km}$ distances), the path loss exponents for ultra high frequency are $n=3.9$ for horizontal and $n=3.7$ for vertical polarizations, and for super high frequency $n=4.6$ for horizontal and $n=$ 4.1 for vertical polarizations. All non-line of sight routes offer larger root mean square delay spread compared with the line of sight scenario. The vertically polarized

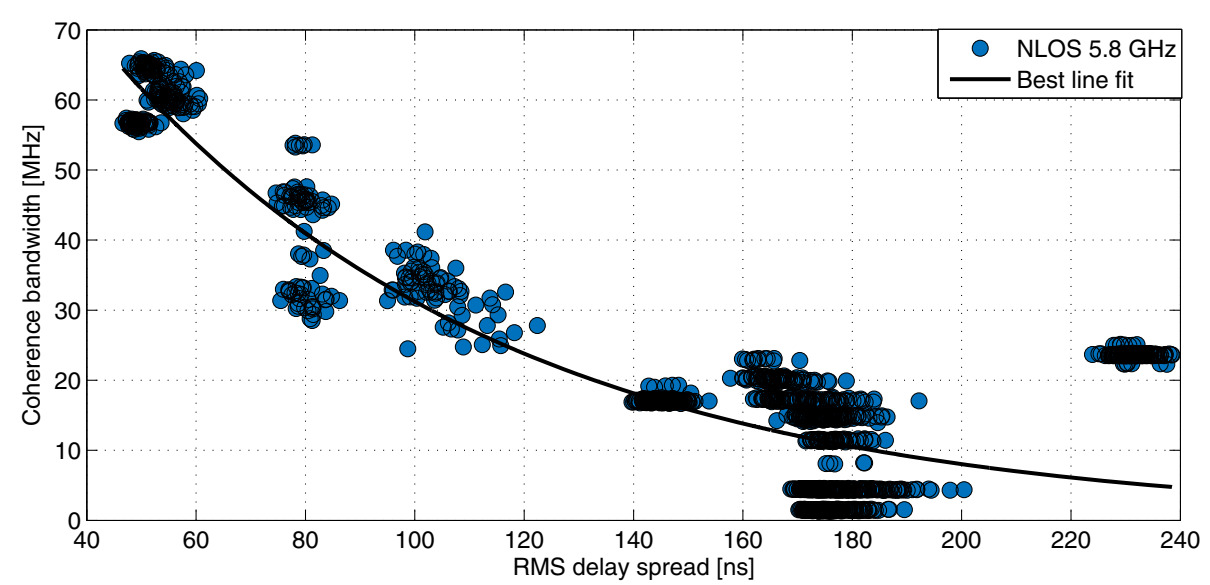

Fig. 13 Coherence bandwidth versus root mean square delay spread for $5.8 \mathrm{GHz}$. Scatter plot of the coherence bandwidth $B_{c, 0.5}$ against the root mean square delay spread in the case of a transmitted signal with $5.8 \mathrm{GHz}$ center frequency in non-line of sight environments 

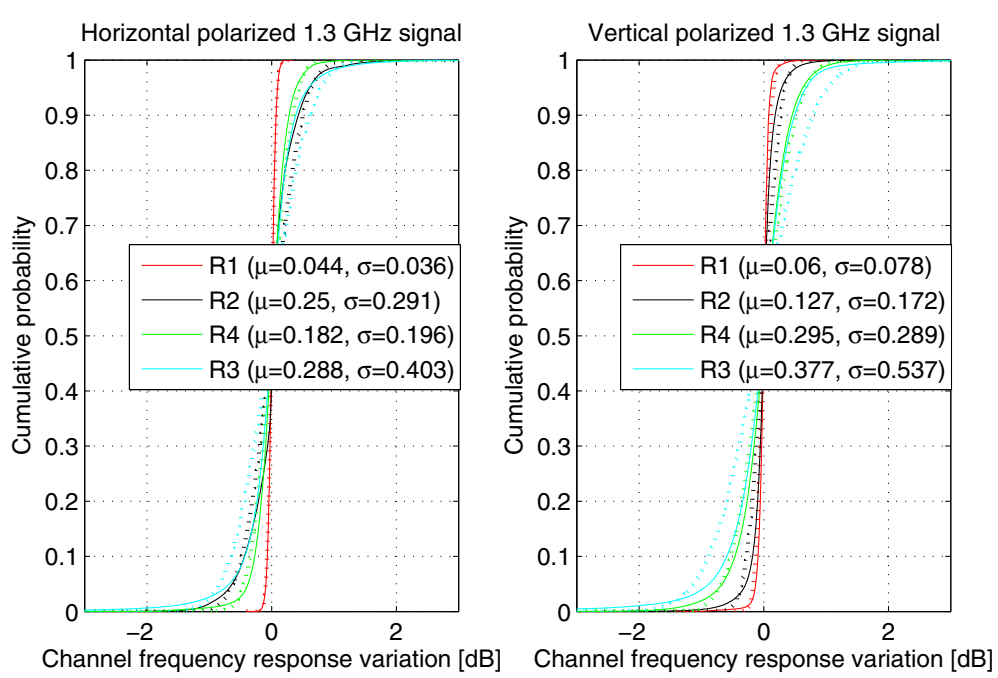

Fig. 14 Cumulative distribution function of channel frequency response variation of $1.3 \mathrm{GHz}$ center frequency for both horizontal and vertical co-polarizations. The curve colored in red, black, blue, and green represent the calculated channel frequency response variation for the first to the fourth routes, respectively. The colored dotted lines represent the normal distribution of the corresponding measured channel frequency response variation values

signal shows higher mean root mean square delay than the horizontally polarized one in the case of ultra high frequency and super high frequency bands for R1, R2, R4 routes. However, an inverse relation was observed in the case of R3 which is explained by depolarization effects caused by the metal roof of the building between the transmitter and the receiver. It was also noticed that the mean root mean square delay spread values increase with the increasing distance between the transmitter and the receiver for all above-tested combinations. Furthermore, the relation between the coherence bandwidth and the root mean square delay spread was investigated. The relation is described by the exponential equation $B_{c, 0.5}=$ $k \cdot e^{-a \sigma_{\tau}}$ where the coherence bandwidth is in megahertz and the root mean square delay spread is in nanoseconds. The channel frequency response variations were also

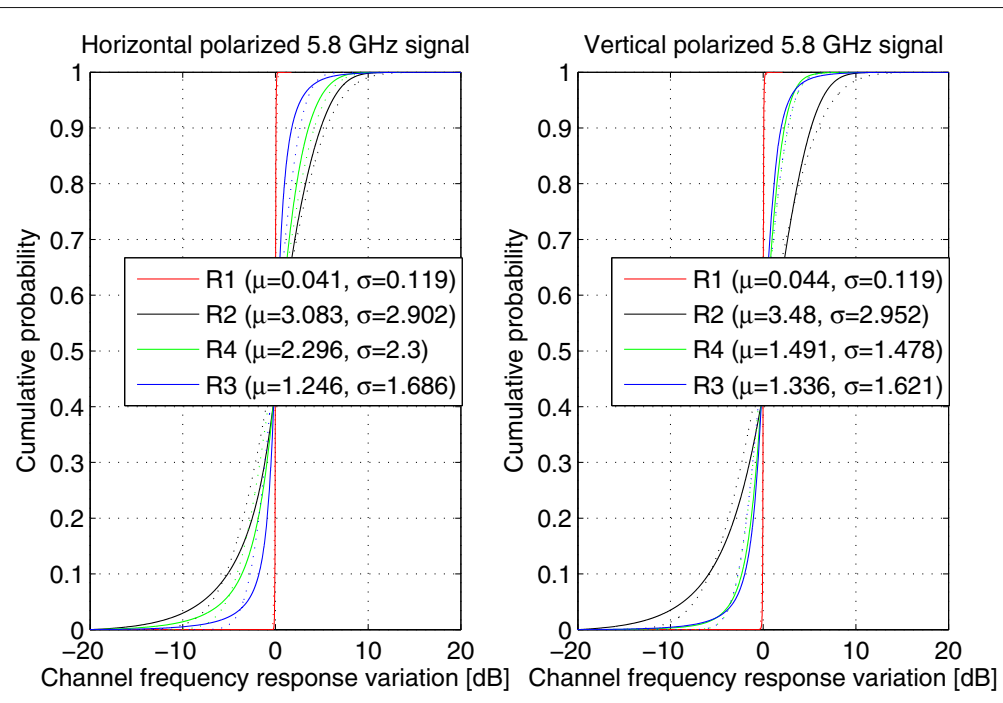

Fig. 15 Cumulative distribution function of channel frequency response variation of $5.8 \mathrm{GHz}$ center frequency for both horizontal and vertical co-polarization. The curve colored in red, black, blue, and green represent the calculated channel frequency response variation for the first to the fourth routes, respectively. The colored dotted lines represent the normal distribution of the corresponding measured channel frequency response variation values 
studied. It was observed that the variation increases with frequency and becomes more critical in the case of nonline of sight scenarios.

\begin{abstract}
Abbreviations
3GPP: 3rd generation partnership project; 5G: 5th generation; ABG: Alpha-beta-gamma; AF: Amplify and forward; AoA: Angle of arrival; AWGN: Additive white gaussian noise; BER: Bit error ratio; BS: Base station; CDF: Cumulative distribution function; CFR: Channel frequency response; CFRV: Channel frequency response variation; Cl: Close-in; CIR: Channel impulse response; D2D: Device to device; DF: Decode and forward; DoA: Delay of arrival; DT: Direct transmission; FM: Frequency modulation; FMCW: Frequency modulated continuous wave; ISI: Inter symbol interference; ITU: International Telecommunication Union; LOS: Line of sight; MIMO: Multiple-input multiple-output; NLOS: Non-line of sight; NR: New radio; OLOS: Obstructed line of sight; RAID: Redundant array of inexpensive disks; RCS: Radar cross section; RF: Radio frequency; RMS: Root mean square; RX: Receiver; SAGE: Space-alternating generalized expectation-maximization; SCC: Spatial correlation coefficient; SHF: Super high frequency; SNR: Signal to noise ratio; TX: Transmitter; UE: User equipment; UHF: Ultra high frequency
\end{abstract}

\section{Acknowledgements}

Not applicable.

\section{Authors' contributions}

EK was involved in analyzing, processing the measured data, deriving the results, and writing the manuscript. JB was responsible for proofreading this work and providing a meaningful feedback. JB, AP, JV, MP, RM, and JH were responsible for carrying out the measurement campaign. All authors read and approved the final manuscript.

\section{Funding}

Research described in this paper was financed by the Czech Ministry of Education within the frame of the National Sustainability Program under grant L01401. It was also financed by the Czech Science Foundation, Project No.

17-27068S. For research, infrastructure of the SIX Center was used.

\section{Availability of data and materials}

The datasets used and/or analysed during the current study are available from the corresponding author on reasonable request.

\section{Competing interests}

The authors declare that they have no competing interests.

\section{Author details}

${ }^{1}$ Department of Radio electronics, Brno University of Technology, Technicka 12, Brno, Czech Republic . RACOM s.r.o, Mírova, Nove Mesto na Morave, Czech Republic

Received: 23 December 2018 Accepted: 3 July 2019

Published online: 23 July 2019

\section{References}

1. C. Yu, K. Doppler, C. B. Ribeiro, O. Tirkkonen, "Resource sharing optimization for device-to-device communication underlaying cellular networks". IEEE Trans. Wirel. Commun. 10(8), 2752-2763 (2011)

2. K. Doppler, M. Rinne, C. Wijting, C. B. Ribeiro, K. Hugl, "Device-to-device communication as an underlay to Ite-advanced networks". IEEE Commun. Mag. 47(12), 42-49 (2009)

3. J. Wang, K. Liu, K. Xiao, C. Chen, W. Wu, V. C. S. Lee, S. H. Son, "Dynamic clustering and cooperative scheduling for vehicle-to-vehicle communication in bidirectional road scenarios". IEEE Trans. Intell. Transp. Syst. 19(6), 1913-1924 (2018)

4. J. Harding, G. Powell, R. Yoon, J. Fikentscher, C. Doyle, D. Sade, M. Lukuc, J. Simons, J. Wang, "Vehicle-to-vehicle communications: Readiness of $\mathrm{v} 2 \mathrm{~V}$ technology for application", (2014)

5. A. H. Kemp, S. K. Barton, "The impact of delay spread on irreducible errors for wideband channels on industrial sites". Wirel. Pers. Commun. 34(3), 307-319 (2005). https://doi.org/10.1007/s11277-005-5230-2
6. W. Fan, I. Carton, J. Ø. Nielsen, K. Olesen, G. F. Pedersen, "Measured wideband characteristics of indoor channels at centimetric and millimetric bands". EURASIP JO. Wirel. Commun. Netw. 2016(1), 58 (2016). https://doi.org/10.1186/s13638-016-0548-x

7. K. Guan, B. Ai, M. L. Nicolás, R. Geise, A. Möller, Z. Zhong, T. Kürner, "On the influence of scattering from traffic signs in vehicle-to-x communications". IEEE Trans. Veh. Technol. 65(8), 5835-5849 (2016)

8. Sun, Shu, et al., in "Propagation path loss models for $5 \mathrm{~g}$ urban micro- and macro-cellular scenarios". 2016 IEEE 83rd Vehicular Technology Conference (VTC Spring). IEEE, 2016

9. J. Chen, X. Yin, L. Tian, N. Zhang, Y. He, X. Cheng, W. Duan, S. Ruiz Boqué, "Measurement-based los/nlos channel modeling for hot-spot urban scenarios in umts networks". Int. J. Antennas Propag. 2014(Article ID 454976) (2014), pp. 12. https://doi.org/10.1155/2014/454976

10. J. Ii, Y. Zhao, C. Tao, B. Ai, "System design and calibration for wideband channel sounding with multiple frequency bands". IEEE Access. 5, 781-793 (2017)

11. R. Nilsson, J. van de Beek, in 2016 IEEE Wireless Communications and Networking Conference. "Channel measurements in an open-pit mine using usrps: $5 \mathrm{~g}$ - expect the unexpected", (Doha, 2016), pp. 1-6. https:// doi.org/10.1109/WCNC.2016.7564672

12. V. Kristem, C. U. Bas, R. Wang, A. F. Molisch, "Outdoor wideband channe measurements and modeling in the $3-18 \mathrm{GHz}$ band". IEEE Trans. Wirel. Commun. 17(7), 4620-4633 (2018)

13. A. Healey, C. H. Bianchi, K. Sivaprasad, in 2000 IEEE-APS Conference on Antennas and Propagation for Wireless Communications (Cat. No.00EX380). "Wideband outdoor channel sounding at $2.4 \mathrm{GHz}$ ", (Waltham, 2000), pp. 95-98. https://doi.org/doi:10.1109/APWC.2000.900150

14. J. Liang, J. Lee, M. Kim, J. Kim, in 2014 International Conference on Information and Communication Technology Convergence (ICTC). "Experimental wideband spatial correlation measurements of low-height mobiles in outdoor urban environments", (Busan, 2014), pp. 854-857. https://doi.org/10.1109/ICTC.2014.6983312

15. W. Wang, T. Jost, U. Fiebig, "A comparison of outdoor-to-indoor wideband propagation at s-band and c-band for ranging". IEEE Trans. Veh. Technol. 64(10), 4411-4421 (2015)

16. E. Suikkanen, L. Hentilä, J. Meinilä, in 2010 Future Network Mobile Summit. "Wideband radio channel measurements around $800 \mathrm{mhz}$ in outdoor to indoor and urban macro scenarios", (Florence, 2010), pp. 1-9

17. X. Nie, J. Zhang, Z. Liu, P. Zhang, Z. Feng, in 2010 IEEE Wireless Communication and Networking Conference. "Experimental investigation of MIMO relay transmission based on wideband outdoor measurements at 2.35 GHz", (Sydney, 2010), pp. 1-6. https://doi.org/10.1109/WCNC.2010.5506459

18. E. Kassem, R. Marsalek, J. Blumenstein, in 2018 25th International Conference on Telecommunications (ICT). "Frequency domain zadoff-chu sounding technique for USRPs", (St. Malo, 2018), pp. 302-306. https://doi. org/10.1109/ICT.2018.8464940

19. T.S.G.R.A.N., 3rd Generation Partnership Project, "User equipment (UE) radio transmission and reception, part 1: range 1 standalone (rel. 15)". 3GPP TS. 38(V1.0.0), 101-1 (2018)

20. T. S. Rappaport, et al., Wireless communications: principles and practice, vol. 2. (Prentice hall PTR, New Jersey, 1996)

21. I.-R. Recommendation, "Guidelines for evaluation of radio transmission technologies for imt-2000 Rec. ITU-RM. 1225,"(1997), pp. 1-60

22. K. Pahlavan, A. H. Levesque, Wireless information networks, vol. 93. (John Wiley \& Sons, 2005)

23. J. M. Molina Garcia Pardo, M. Lienard, P. Degauque, "Propagation in tunnels: Experimental investigations and channel modeling in a wide frequency band for MIMO applications". EURASIP J. Wirel. Commun. Netw. 2009(1), 560571 (2009)

24. M.-G. Di Benedetto (ed.), UWB communication systems: a comprehensive overview, vol. 5 (Hindawi Publishing Corporation, 2006)

25. D. C. Stone, "Application of median filtering to noisy data". Can. J. Chem. 73(10), 1573-1581 (1995)

26. R. G. Vaughan, "Signals in mobile communications: A review". IEEE Trans. Veh. Technol. 35(4), 133-145 (1986)

27. W. C. Y. Lee, Y. Yeh, "Polarization diversity system for mobile radio". IEEE Trans. Commun. 20(5), 912-923 (1972)

28. G. R. MacCartney, T. S. Rappaport, "Rural macrocell path loss models for millimeter wave wireless communications". IEEE J. Sel. Areas Commun. 35(7), 1663-1677 (2017) 
29. H. Hashemi, D. Tholl, "Statistical modeling and simulation of the rms delay spread of indoor radio propagation channels". IEEE Trans. Veh. Technol. 43(1), 110-120 (1994)

30. T. S. Rappaport, G. R. MacCartney, M. K. Samimi, S. Sun, "Wideband millimeter-wave propagation measurements and channel models for future wireless communication system design". IEEE Trans. Commun. 63(9), 3029-3056 (2015)

31. S. Sangodoyin, S. Niranjayan, A. F. Molisch, "A measurement-based model for outdoor near-ground ultrawideband channels". IEEE Trans. Antennas Propag. 64(2), 740-751 (2016)

32. L. Rubio, J. Reig, H. Fernández, M. R. P. Vicent, "Experimental uwb propagation channel path loss and time-dispersion characterization in a laboratory environment". Int. J. Antennas Propag. 2013, 1-7 (2013)

33. S. M. Yano, in Vehicular Technology Conference. IEEE 55th Vehicular Technology Conference. "Investigating the ultra-wideband indoor wireless channel", vol. 3 (IEEE, VTC Spring, 2002), pp. 1200-1204

34. Y. Wang, W. Lu, H. Zhu, "Propagation characteristics of the Ite indoor radio channel with persons at $2.6 \mathrm{GHz}$ ". IEEE Antennas Wirel. Propag. Lett. 12, 991-994 (2013)

35. A. M. Tonello, F. Versolatto, B. Bejar, "A top-down random generator for the in-home plc channel". 2011 IEEE Global Telecommun. Confer. GLOBECOM. 2011, 1-5 (2011)

\section{Publisher's Note}

Springer Nature remains neutral with regard to jurisdictional claims in published maps and institutional affiliations.

\section{Submit your manuscript to a SpringerOpen ${ }^{\circ}$ journal and benefit from:}

- Convenient online submission

- Rigorous peer review

- Open access: articles freely available online

- High visibility within the field

- Retaining the copyright to your article

Submit your next manuscript at $>$ springeropen.com 